\title{
Wet age-related macular degeneration combined with branch retinal vein occlusion
}

\author{
Ioannis Mallias, Panagiota Mylova, Anastasia Tassiopoulou \\ Laser Plus Eye, Nea Smyrni, Athens, Greece
}

\begin{abstract}
To disclose a case of wet age-related macular degeneration (wet AMD) combined with branch retinal vein occlusion (BRVO). The prognosis and the treatment are discussed. A 77-year-old woman presented with wet AMD of the left eye. The patient was treated with intravitreal injection of ranibizumab of the left eye with improvement of visual acuity and resolution of intra-retinal and sub-retinal fluid. One year after the last injection the patient developed a BRVO of the same eye. A flame shaped haemorrhage was observed, and OCT examination showed cystic macular oedema. Fluorescein angiography showed that there was an active lesion of choroidal neovascularisation (CNV), areas of ischaemia, delayed filling and emptying of the affected vein, micro aneurisms, and telangiectatic changes. The co-existence of BRVO and active WET AMD was documented by fluorescein angiography. The co-existence of both diseases has not been described in the literature until today. This co-existence makes the prognosis worse and diminishes the patient's visual acuity.
\end{abstract}

KEY WORDS: branch retinal vein occlusion, wet age-related macular degeneration

Ophthalmol J 2017; Vol. 2, No. 3, 87-90

\section{INTRODUCTION}

It is common knowledge that macular degeneration is diagnosed as either dry (non-neovascular) or wet (neovascular) [1]. The majority of patients present with dry macular degeneration (DRY AMD). In about $10 \%$ of all patients diagnosed with macular degeneration, the disease progresses to wet type (wet AMD). Some of the risk factors for wet AMD are age $[1,2]$, smoking [3, 4], and genetic susceptibility [5]. Other risk factors are cardiovascular disease, hypertension, female gender, white race [6], hypercholesterolaemia, obesity, hyperopia [7], and family history.

In order to observe and evaluate the progression of AMD (both dry and wet), retinoscopy with full pupil dilation is necessary. Optical Coherence Tomography (OCT) offers a high-resolution, non-invasive, optical cross-sectional method that utilises low-coherence interferometry [8]. OCT is a valua- ble diagnostic tool that helps monitor and evaluate the progression of the disease with accuracy.

Although there is no current treatment for dry AMD, when it comes to wet AMD there are several options. Macular photocoagulation was studied in 1980s for limiting damage caused by choroidal neovascular lesions $[9,10]$. Photodynamic therapy was also used in the late 1990s. The most up-todate treatment for wet AMD is probably anti-vascular endothelial growth factor (anti-VEGF) injections. Substances such as ranibizumab, bevacizum$\mathrm{ab}$, and aflibercept have been employed to reduce the proliferation of abnormal leaky blood vessels grown from the choroidal layer through Bruch's membrane, into the retinal layers.

Branch retinal vein occlusion (BRVO) is a venous occlusion at any branch of the central retinal vein. It is classified according to the anatomical location as major or macular. Major BRVO refers 


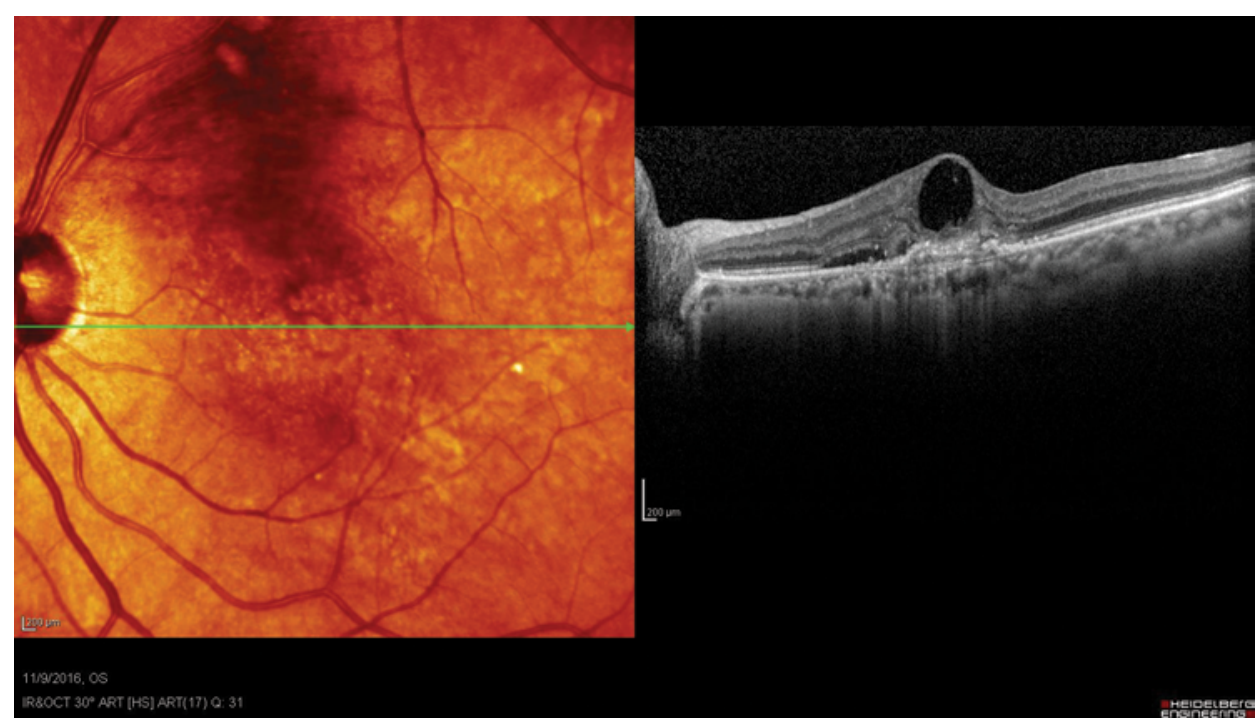

FIGURE 1. OCT image of the patient during a routine visit

to occlusion of a retinal vein that drains one of the quadrants. Macular BRVO refers to occlusion of a venule within the macula [11]. Some risk factors leading to BRVO are age, hypertension, hyperlipidaemia, ocular hypertension, and glaucoma [12,13].

When treating BRVO, the aimed treatment is to prevent the complications that cause vision loss, such as macular oedema, macular ischaemia, and neovascularisation. BRVO can be treated with photocoagulation [14, 15], steroid treatment [16], and/or anti-VEGF injections [17-19].

\section{CASE REPORT}

A 77-year-old female came to the office on a scheduled appointment. The patient's visual acuity was $20 / 20$ in the right eye and 20/400 in the left eye. She was already diagnosed with wet AMD in the left eye and had already received eight anti-VEGF injections.

On the left eye, a haemorrhage was observed with fundoscopy, which referred to BRVO. An OCT examination was performed which showed cystic macular oedema, sub-retinal fluid, and an area of hyper-reflectivity at the ISOS junction of the left eye (Fig. 1).

From the fundus image, we suspected that the case of wet AMD was complicated by BRVO. A fluorescein angiography was performed.

Blocked fluorescence was observed due to the retinal haemorrhages seen through all phases of the angiography. There was delayed filling and emptying of the affected vein, dilated capillaries, micro- -aneurisms, and telangiectatic changes. Areas of capillary closure were presented on the upper left quadrant. Late staining and leakage from the affected vein was observed. In the subfoveal area, there was staining due to old CNV lesions. In the inferotemporal area of the fovea, there was a spot of hyper-fluorescence, which corresponded to an area of active choroidal neovascularisation.

In Figure 2 and 3 the early and late times of the test are depicted.

Fluorescein angiography proved the existence of an active choroidal neovascularisation combined with BRVO. We decided to treat the patient with a ranibizumab injection.

After the injection, the patient was prescribed oxifloxacin four times daily for a week in order to avoid the risk of ocular infection. Three days after the injection another OCT was performed, which showed improvement of both macular oedema (Fig. 4).

One month after the anti-VEGF injection, the patient came for a follow up. A measurement of Best Corrected Visual Acuity (BCVA) was performed as well as OCT imaging (Fig. 5).

Unfortunately, visual acuity remained 20/400. OCT examination showed complete resolution of the cystoid macular oedema and sub-retinal fluid. Fundus examination showed resolution of the haemorrhages of the BRVO as well.

\section{DISCUSSION}

Despite the fact that the patient responded very well to the treatment, visual acuity did not improve 
at all. This was attributed to the fact that the big cystic spaces filled with fluid, even though they dried out with the anti-VEGF injection, caused permanent damage to the intra-retinal network. This damage contributed to poor visual acuity.

To our knowledge there has not been any other report on the literature of wet AMD co-existing with BRVO. This was probably the most aggravating factor of the remaining poor visual acuity.

As referred in the literature, wet AMD and BRVO have several risk factors in common, such as hypertension and cardiovascular disease. In the re-

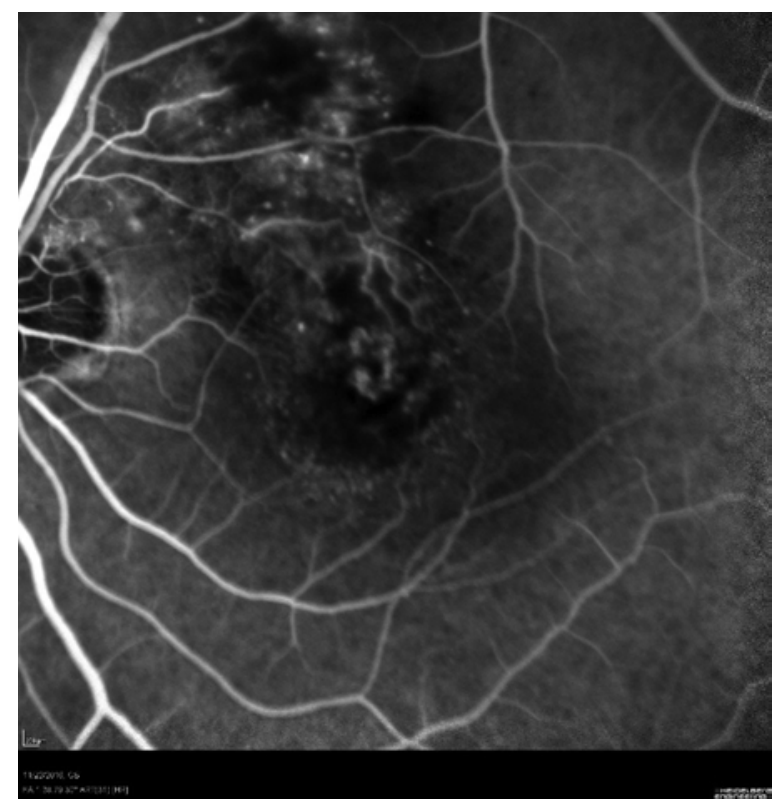

FIGURE 2. Early times of fluorescein angiography ported case, the patient was already diagnosed with hypertension and is now under treatment.

What should also be mentioned is that the patient is diabetic. That plays an important role in the quality of the patient's blood vessels all over her body and, in this case, in the retina. Diabetes can contribute to BRVO, usually by causing high blood pressure [20]. Moreover, there are studies [21] linking diabetes to severe wet AMD.

Concluding, when combining diabetes and hypertension (that may or may not have derived from diabetes), a possible explanation occurs for the co-ex-

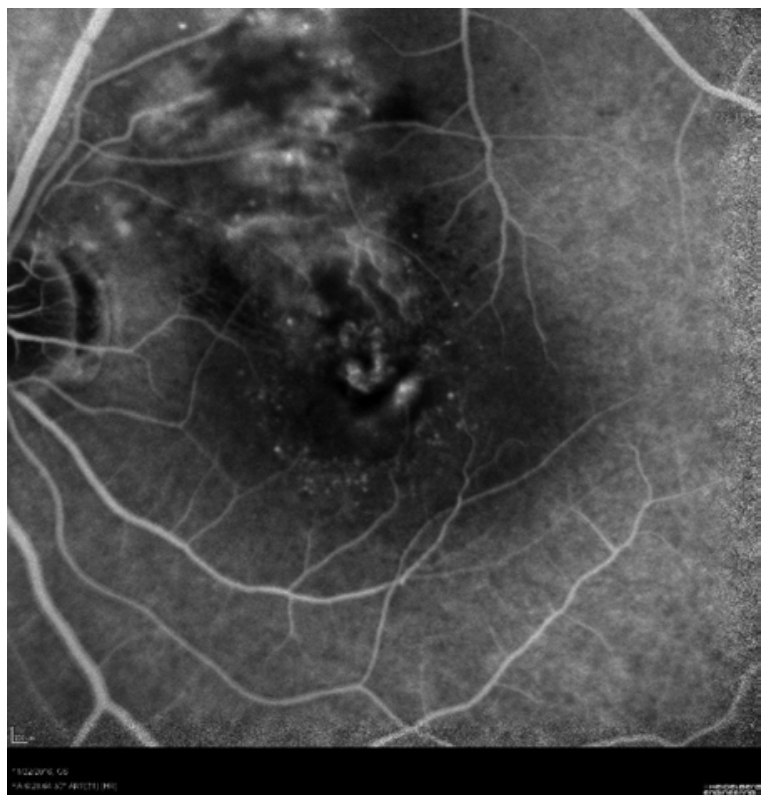

FIGURE 3. Late times of fluorescein angiography

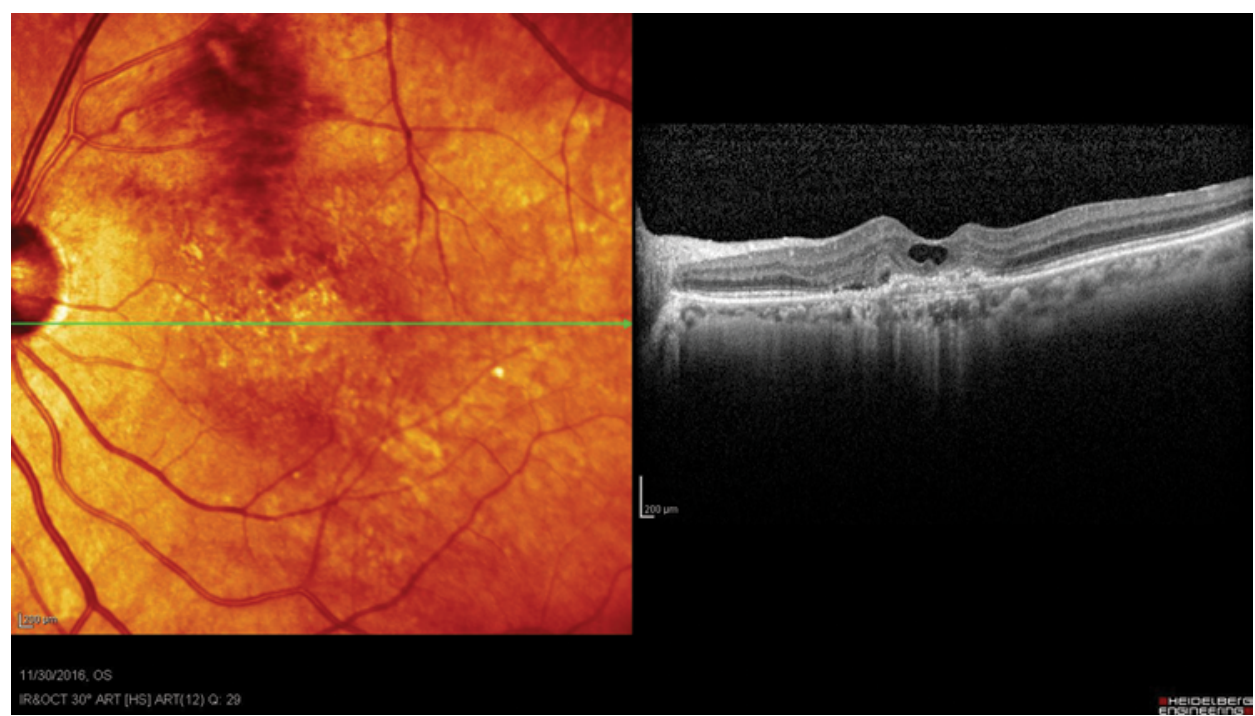

FIGURE 4. OCT image of the patient 3 days after anti-VEGF injection 


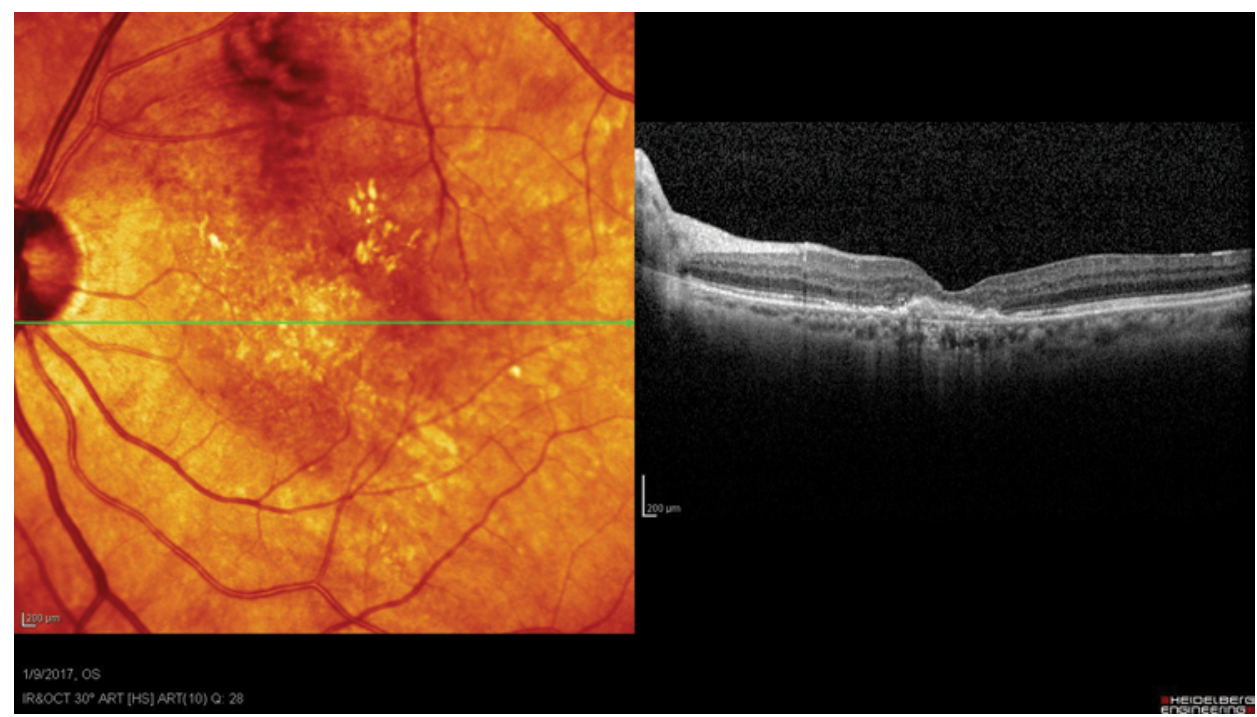

FIGURE 5. OCT image, one month after anti-VEGF injection

istence of BRVO and wet AMD. What should be noted is that patients who already know that they have hypertension and/or diabetes should check regularly with their ophthalmologist for a thorough examination that also includes OCT imaging. In this way there is a high possibility to control and prevent any pathological changes in the fundus from becoming permanent and to lead to irreversible loss of vision.

\section{REFERENCES}

1. Klein $R$, Klein $B$, Linton K. Prevalence of age-related aaculopathy. Ophthalmology. 1992; 99(6): 933-943, doi: 10.1016/s01616420(92)31871-8.

2. Leibowitz $H$, Krueger $D E$, Maunder $L R$, et al. The Framingham Eye Study Monograph: An ophthalmological and epidemiological study of cataract, glaucoma, diabetic retinopathy, macular degeneration and visual acuity in a general population of 2631 adults. Surv Ophthalmol. 1980; 24: 335-610.

3. Clemons TE, Milton RC, Klein R, et al. Age-Related Eye Disease Study Research Group. Risk factors for the incidence of Advanced Age-Related Macular Degeneration in the Age-Related Eye Disease Study (AREDS) AREDS report no. 19. Ophthalmology. 2005; 112(4): 533-539, doi: 10.1016/j.ophtha.2004.10.047, indexed in Pubmed: 15808240.

4. Khan JC, Thurlby DA, Shahid H, et al. Genetic Factors in AMD Study. Smoking and age related macular degeneration: the number of pack years of cigarette smoking is a major determinant of risk for both geographic atrophy and choroidal neovascularisation. Br J Ophthalmol. 2006; 90(1): 75-80, doi: 10.1136/bjo.2005.073643, indexed in Pubmed: 16361672.

5. Smailhodzic D, Muether PS, Chen J, et al. Cumulative effect of risk alleles in CFH, ARMS2, and VEGFA on the response to ranibizumab treatment in age-related macular degeneration. Ophthalmology. 2012; 119(11): 2304-2311, doi: 10.1016/j.ophtha.2012.05.040, indexed in Pubmed: 22840423

6. Friedman DS, Katz J, Bressler NM, et al. Racial differences in the prevalence of age-related macular degeneration: the Baltimore Eye Survey. Ophthalmology. 1999; 106(6): 1049-1055, doi: 10.1016/ S0161-6420(99)90267-1, indexed in Pubmed: 10366070.

7. Sandberg MA, Tolentino MJ, Miller S, et al. Hyperopia and neovascularization in age-related macular degeneration. Ophthalmology. 1993; 100(7): 1009-1013, indexed in Pubmed: 7686644.

8. Huang D, Swanson EA, Lin CP, et al. Optical coherence tomography. Science. 1991; 254(5035): 1178-1181, indexed in Pubmed: 1957169.
9. Argon laser photocoagulation for senile macular degeneration. Results of a randomized clinical trial. Arch Ophthalmol. 1982; 100(6): 912-918, indexed in Pubmed: 7046707.

10. Argon laser photocoagulation for neovascular maculopathy. Threeyear results from randomized clinical trials. Macular Photocoagulation Study Group. Arch Ophthalmol. 1986; 104(5): 694-701, indexed in Pubmed: 2423061.

11. Hayreh SS. Ocular vascular occlusive disorders: natural history of visual outcome. Prog Retin Eye Res. 2014; 41: 1-25, doi: 10.1016/j. preteyeres.2014.04.001, indexed in Pubmed: 24769221.

12. Risk factors for branch retinal vein occlusion. The Eye Disease Case-control Study Group. Am J Ophthalmol. 1993; 116(3): 286-296, indexed in Pubmed: 8357052.

13. Kolar P. Risk factors for central and branch retinal vein occlusion: a meta-analysis of published clinical data. J Ophthalmol. 2014; 2014: 724780, doi: 10.1155/2014/724780, indexed in Pubmed: 25009743.

14. Argon laser photocoagulation for macular edema in branch vein occlusion. The Branch Vein Occlusion Study Group. Am J Ophthalmol. 1984; 98(3): 271-282, indexed in Pubmed: 6383055.

15. Argon Laser Scatter Photocoagulation for Prevention of Neovascularization and Vitreous Hemorrhage in Branch Vein Occlusion. Archives of Ophthalmology. 1986; 104(1): 34, doi: 10.1001/archopht.1986.01050130044017.

16. McAllister IL, Vijayasekaran S, Chen SD, et al. Effect of triamcinolone acetonide on vascular endothelial growth factor and occludin levels in branch retinal vein occlusion. Am J Ophthalmol. 2009; 147(5): 838-46, 846.e1, doi: 10.1016/j.ajo.2008.12.006, indexed in Pubmed: 19211093.

17. Yoshimura T, Sonoda Kh, Sugahara M, et al. Comprehensive analysis of inflammatory immune mediators in vitreoretinal diseases. PLoS One. 2009; 4(12): e8158, doi: 10.1371/journal.pone.0008158, indexed in Pubmed: 19997642.

18. Noma $H$, Funatsu $H$, Yamasaki $M$, et al. Pathogenesis of macular edema with branch retinal vein occlusion and intraocular levels of vascular endothelial growth factor and interleukin-6. Am J Ophthalmol. 2005; 140(2): 256-261, doi: 10.1016/j.ajo.2005.03.003, indexed in Pubmed: 16086947.

19. Karia N. Retinal vein occlusion: pathophysiology and treatment options. Clin Ophthalmol. 2010; 4: 809-816, indexed in Pubmed: 20689798.

20. Kolar P. Risk factors for central and branch retinal vein occlusion: a meta-analysis of published clinical data. J Ophthalmol. 2014; 2014: 724780, doi: 10.1155/2014/724780, indexed in Pubmed: 25009743.

21. Chen $X$, Rong SS, Xu 0 , et al. Diabetes mellitus and risk of age-related macular degeneration: a systematic review and meta-analysis. PLoS One. 2014; 9(9): e108196, doi: 10.1371/journal.pone.0108196, indexed in Pubmed: 25238063. 